\title{
Comparison of skeletal, dental and soft tissue changes in young adults with Class II malocclusion, treated either by camouflage, fixed functional appliance or orthognathic surgery-A prospective study on Indian subjects
}

\author{
Anjana Shetty ${ }^{1}$, Anand Patil ${ }^{2}$, Sanjay Ganeshkar ${ }^{2}$ \\ ${ }^{1}$ Department of Orthodontics and Dentofacial Orthopaedics, AECS Maaruthi Dental College, Banglore, India \\ ${ }^{2}$ Department of Orthodontics and Dentofacial Orthopaedics, SDM College of Dental Sciences, Dharwad, India \\ Email: anjanashetty@yahoo.com
}

Received 1 October 2012; revised 3 November 2012; accepted 15 November 2012

\begin{abstract}
Introduction: Borderline Class II malocclusion due to deficient mandible can be treated either by orthodontic camouflage, fixed functional appliances or by orthodontics followed by surgical mandibular advancement. Methodology: A prospective study was designed on young adults with Class II malocclusion on account of a deficient mandible. A total of 45 subjects were divided into three groups of 15 individuals each. The patients were treated either by camouflage, fixed functional appliances or by orthognathic surgery. Pre and post treatment cephalograms were used to assess the skeletal, dental and soft tissue changes. Pre and post treatment profile photographs were assessed on a Visual Analogue Scale (VAS) by orthodontists, oral surgeons and laypersons. Results: Each group achieved a reduction in facial convexity, but the results obtained from the surgical group were more pronounced than the camouflage and the fixed functional group. Conclusion: The reduction in convexity in the camouflage group was by retracting the upper anteriors, which increases the nasolabial angle. In the fixed functional appliance a combination of skeletal and dentoalveolar changes can be observed. However the most appropriate reduction in profile convexity can be obtained by combined orthodontic and surgical treatment of malocclusion.
\end{abstract}

Keywords: Class II Malocclulsion; Camouflage; Fixed Function Appliance; Orthognathic Surgery

\section{INTRODUCTION}

Class II malocclusions are of interest to the practicing orthodontists since they constitute a significant percentage of the cases they treat and the interest of the ortho- dontist in adult orthodontics has also increased.

Class II malocclusion is a common disharmony that has been studied in many different populations. Because excessive overjet is easily recognized, Class II Division 1 malocclusion is of great concern to patients. Class II malocclusion may result from numerous combinations of skeletal and dental components. Mandibular retrognathism is present in most patients with a skeletal Class II malocclusion. In Indian population there's a varied opinion about individuals with a convex profile. This makes decision making a challenge to the attending orthodontist. With so many treatment options available for Class II malocclusions cases choosing the right treatment modality should not only be evidence-based but also based on the visual prespective. This becomes even more challenging in boderline Class II malocclusion hence this study.

There are three possible approaches for the treatment of moderate Class II malocclusions (borderline cases) in non-growing patients $[1,2]$ :

1) Tooth movement to compensate for the jaw discrepancy — camouflage orthodontics $[3,4]$;

2) Fixed functional orthopedic appliance [5-9];

3) Orthognathic surgery, which involves mandibular advancement $[10,11]$.

The first technique involves orthodontic measures alone to achieve a dental compensation of the malocclusion $[3,4]$.

The second treatment alternative involves the use of a fixed functional orthopedic appliance, whereby residual growth is harnessed, stimulating remodelling processes in the temporomandibular joint area, which combined with dentoalveolar effects, achieve successful bite correction. It has a stimulatory effect on the temporomandibular joint, and hence is considered as an useful orthopedic tool for non-surgical, non-extraction treatment in borderline Class II adults [5-9]. 
The third option is the combined orthodontic and surgical treatment of the jaw discrepancy. The focus is advancement of the mandible to correct the mandibular deficiency $[9,10]$.

Patients with nearly identical mandibular deficiency and Class II malocclusion were treated by either one of the three treatment options. Hence a detailed study of every individual patient and what treatment option will bring about the desired results should be carefully pondered over.

The overall aim of any of the treatment options available is to enhance both, the dental and facial esthetics $[12,13]$. To rate this, a Visual Analogue Scale was used to score the pre and post-treatment attractiveness of the patients treated with profile photographs. This was scored by Orthodontists, Oral surgeons and laypersons, to assess how individuals from different views appreciate the treatment outcome in all the three groups.

\section{METHODOLOGY}

A study was designed which included a total of 45 young adults from an Indian population with boderline skeletal Class II malocclusion, on account of a retrognathic mandible. Out of the total sample pool, 15 individuals underwent camouflage orthodontics ( $\mathrm{C}$ group), 15 were treated by fixed mechanotherapy along with fixed functional appliances (FFA group) and 15 underwent orthognathic surgery, which involved surgical advancement of the mandible only (OS group). All the patients selected were treated by pre-adjusted edgewise appliance (0.022" slot) with MBT prescription. In the cases treated by fixed functional appliances a moderate labial root torque was incorporated on a $0.019 " \times 0.025^{\prime \prime}$ stainless steel in addition to the built-in 6 degree labial root torque for the lower anteriors to prevent lower incisor proclination $[7-9,14,15]$.

All the individuals had an overjet exceeding $6 \mathrm{~mm}$ and molar relationship ranging from end-on to full cusp Class II. The samples growth pattern ranged from being normal to mild hyperdivergent jaw bases.

Depending on the diagnosis and the patient's treatment needs, the cases were treated by either one of the treatment options.

Samples in each group were randomly selected to rate the pre and post-treatment changes in profile that could be observed. The scoring was done by Orthodontists, Oral surgeons and laypersons to see how differently or similarly the treatment outcome was perceived by different sectors of people.

\subsection{Selection Criteria}

The samples selected had moderate skeletal Class II malocclusions and were based on the following criteria:
1) Clinical parameters included,

a) Convex profile;

b) Average growth pattern of mandible.

2) Cephalometrically,

a) ANB angle ranging from 4 - 8 degrees;

b) Retrognathic mandible.

3) Dental pattern,

a) Increased overjet and overbite;

b) Class II division 1 malocclusion.

4) Age ranging from 16 to 25 years,

5) Patients with gross facial asymmetry were excluded from the study.

\subsection{Cephalometric Assessment}

A single operator performed the tracings in a standardized manner to avoid errors due to inter-operator variability. The hard and soft tissue landmarks, reference planes and angular measurements, as defined by Alexander Jacobson, Thomas Rakosi and Athanasious E. Athanasious were used; i.e. a composite cephalometric analysis was conducted.

A) Measurements indicating skeletal antero-posterior relationship:

1) Angle ANB (Steiner) - It is the difference between angles SNA and SNB. It provides a general idea of anteroposterior discrepancy of the maxilla to mandibular apical bases.

2) Angle NA-Pg (Down)-It measures the degree of maxillary basal arch at its anterior limit (point A) relative to total facial profile.

3) A perpendicular to $\mathrm{B}$ perpendicular on $\mathrm{FH}$ plane and Wits appraisal-Are adjunctive diagnostics in assessing the extent of anteroposterior discrepancy.

B) Measurements indicating maxillary size and placement:

1) Angle SNA (Steiner) and A perpendicular to $\mathrm{N}$ perpendicular on FH plane-Determines the maxillary apical base position with respect to " $\mathrm{N}$ " point.

2) $\mathrm{S}$ perpendicular to Ptm perpendicular on nasal floor-It gives the placement of posterior limit of the maxilla with respect to $\mathrm{S}$ point.

C) Measurements indicating mandibular size and placement:

1) Angle SNB (Steiner's) and B perpendicular to $N$ perpendicular on FH plane-Determines the mandibular apical base position with respect to $\mathrm{N}$ point.

2) Linear measurement from Gonion to PogonionGives the length of mandibular corpus.

3) Saddle angle and linear measurement from Sella to Articulare (Posterior cranial base length)-Gives the placement of the mandibular condyle with respect to Sella.

4) Posterior cranial base (PCB) height is the linear measurement from the Sella to Articulare. 
D) Measurements indicating vertical skeletal relations:

1) Anterior facial height $(\mathrm{N}-\mathrm{Me})$ and Posterior face height (S-Go) are linear measurements used to determine the Jarabak ratio $\{(S-G o: N-M e)$ is the ratio of the posterior to anterior face height $\}$.

2) Angle SN to Go-Gn-Relates the lower border of the mandible to the anterior cranial base.

3) Basal plane angle-Is the angle between maxillary and mandibular planes and relates the lower border of the mandible to the maxillary plane.

4) Frankfort mandibular plane angle (Tweed) - It is the angle between Go-Me to FH plane and relates the lower border of the mandible to the $\mathrm{FH}$ plane.

E) Measurements indicative of dentoalveolar changes:

1) Upper incisor to $\mathrm{SN}$-Angular measurement and Upper incisor to NA-Both angular and linear measurements-Gives the inclination and the distance of the upper incisor tip to the reference planes.

2) Lower incisor to Mandibular Plane-Gives the inclination and the distance of the lower incisor tip to the reference planes.

F) Measurements indicative of soft tissue relations:

1) Soft tissue facial angle N'-Pg to $\mathrm{FH}$-indicates the relative position of the chin with respect to the upper face.

2) Nasolabial angle-It is the angle between the tangents to the Columella and upper lip and indicates lip protrusion.

3) Mentolabial angle - It is the angle between tangents to Labrale inferius and Pg' and is an indicator of lip protrusion.

The pre-treatment and post-treatment profile photographs of 10 random cases from each group was selected i.e. patients treated either by camouflage orthodontics alone, fixed appliance along with Fixed Functional Appliance and those treated by orthognathic surgery (bilateral sagittal split osteotomy) were scored on Visual Analouge Scale (VAS), by 10 orthodontists, 10 oral surgeons and 10 laypersons. The VAS has been used to assess dentofacial esthetic assessment [13]. The scoring ranged from 0 to 10 , with 0 being the most attractive and 10 as the least attractive treatment outcome.

Measurements indicating skeletal antero-posterior relationship, maxillary size and placement, mandibular size and placement, vertical skeletal relations, dentoalveolar changes and measurements indicative of soft tissue relations were analysed. The results were subjected to ANOVA and Tukey's Honestly Significant Difference (HSD) Test. The quantative variables were compared using MannWhitney test.

\section{RESULTS}

The comparison of skeletal parameters between treatment groups, which includes changes in the sagittal rela- tions, the position of the two jaw bases and the vertical positions have been included. One-way ANOVA test showed that there were no statistically significant differences between the treatment groups to start with. When the pre and post-treatment outcomes were compared, there was a highly significant change seen with the treatment outcomes.

Following which, pair wise comparison of the three groups (C, FFA and OS) with respect to the pre and post-treatment values were compared by Tukey's Honest Significant Difference (HSD) test procedure, to compare each group against the other and to find out, which among the three groups had significant changes when compared with each other.

1) Changes in the inter-arch sagittal skeletal relationship: (Tables 1(a) and (b))

C group: All the reading showed that there was no statistically significant change seen in the skeletal parameters in the sagittal aspect which included the ANB angle, Wits appraisal and N-A-Pg angle.

FFA group: There was a statistically significant change seen with respect to the ANB angle (mean diff. 1.6 ${ }^{\circ}$ ), whereas the other measurements showed no changes.

OS group: All the measurements showed a highly significant change, with ANB (mean diff. 3.9 $)$, Wits appraisal (mean diff. $5.8 \mathrm{~mm}$ ), and N-A-Pg angle (mean diff. $\left.5.8^{\circ}\right)$.

2) Changes in the size of the mandible: (Tables $\mathbf{2}$ and $\mathbf{3}$ )

$\mathrm{C}$ group: There was no significant change observed in the size and position of the mandible at the end of fixed mechanotherapy, since the treatment was carried out entirely by changes in the dentoalveolar region.

FFA group: Mandibular corpus length (mean diff. -1.40 $\mathrm{mm})$ showed a highly significant change $(\mathrm{p}=0.0028)$. This could be attributed to the stimulation of residual growth in these young adults.

OS group: SNB angle (mean diff. $-3.6^{\circ}$ ) showed a statistically significant change $(\mathrm{p}=0.0001)$, while there was also a highly significant increase $(p=0.0000)$ in the mandibular corpus length (mean diff. $-6.1 \mathrm{~mm}$ ). It can be inferred that the sagittal skeletal correction was on account of the surgical advancement of the mandible.

3) Changes in the vertical skeletal proportions: (Tables 4(a) and (b))

$\mathrm{C}$ group: Insignificant changes in the vertical jaw relationship was seen when the pre and post-treatment parameters were compared.

FFA group: There were no changes seen in this group either, indicating the divergence of jaw bases was not altered.

OS group: FMA angle was the only parameter which had increased (mean diff. $-2.1^{\circ}$ ), it showed a statistically significant change $(\mathrm{p}=0.0301)$, which indicated that there, was some amount of the mandible rotating downward and backwards, although not clinically significant. 
Table 1. (a) Comparison of three groups (C, FFA and OS) with respect to pre, post and difference scores of ANB angle by One way ANOVA test; (b) Pair wise comparison of three groups (C, FFA and surgical) with respect to pre, post and difference scores of ANB angle by Tukey's Honest Significant (HSD) Difference test procedure.

(a)

\begin{tabular}{|c|c|c|c|c|c|c|}
\hline Variable & Source of variation & Degrees of freedom & Sum of squares & Mean sum of squares & F-value & p-value \\
\hline \multirow{3}{*}{ Pre treatment } & Between groups & 2 & 2.500 & 1.2500 & 0.3679 & 0.6944 \\
\hline & Within groups & 42 & 142.700 & 3.3976 & & \\
\hline & Total & 44 & 145.200 & & & \\
\hline \multirow{3}{*}{ Post treatment } & Between groups & 2 & 46.800 & 23.4000 & 6.1272 & $0.0046^{* *}$ \\
\hline & Within groups & 42 & 160.400 & 3.8190 & & \\
\hline & Total & 44 & 207.200 & & & \\
\hline \multirow{3}{*}{ Difference } & Between groups & 2 & 70.300 & 35.1500 & 9.7317 & $0.0003^{* *}$ \\
\hline & Within groups & 42 & 151.700 & 3.6119 & & \\
\hline & Total & 44 & 222.000 & & & \\
\hline
\end{tabular}

(b)

\begin{tabular}{ccccc}
\hline Treatment & Group & C & FFA & OS \\
\hline \multirow{4}{*}{ Pre treatment } & Mean & 5.8667 & 5.8667 & 6.3667 \\
& C & - & & \\
& FFA & 1.0000 & - & - \\
& OS & 0.7396 & 0.7396 & 2.4667 \\
Post treatment & Mean & 4.8667 & 4.2667 & \\
& C & - & & - \\
& FFA & 0.6801 & - & 3.9000 \\
Difference & OS & $0.0047^{* *}$ & $0.0404^{*}$ & \\
& Mean & 1.0000 & 1.6000 & - \\
\hline
\end{tabular}

Table 2. (a) Comparison C, FFA and OS with respect to pre, post and difference scores of SNB angle by One-Way ANOVA test; (b) Pair wise comparison of C, FFA and OS with respect to pre, post and difference scores of SNB angle by Tukey's Honest Significant (HSD) Difference test procedure.

(a)

\begin{tabular}{|c|c|c|c|c|c|c|}
\hline Variable & Source of variation & Degrees of freedom & Sum of squares & Mean sum of squares & F-value & p-value \\
\hline \multirow{3}{*}{ Pre treatment } & Between groups & 2 & 37.544 & 18.77 & 1.28 & 0.2869 \\
\hline & Within groups & 42 & 612.90 & 14.59 & & \\
\hline & Total & 44 & 650.44 & & & \\
\hline \multirow{3}{*}{ Post treatment } & Between groups & 2 & 20.311 & 10.15 & 0.82 & 0.4441 \\
\hline & Within groups & 42 & 515.33 & 12.269 & & \\
\hline & Total & 44 & 535.64 & & & \\
\hline \multirow{3}{*}{ Difference } & Between groups & 2 & 104.67 & 52.33 & 8.10 & $0.0011^{* *}$ \\
\hline & Within groups & 42 & 271.30 & 6.459 & & \\
\hline & Total & 44 & 35.977 & & & \\
\hline
\end{tabular}

(b)

\begin{tabular}{ccccc}
\hline Treatment & Group & C & FFA & OS \\
\hline Pre treatment & Mean & 75.933 & 76.5330 & 74.3670 \\
& C & - & & \\
& FFA & 0.903 & - & - \\
Post treatment & OS & 0.505 & 0.2771 & 78.0000 \\
& Mean & 76.400 & 76.8670 & \\
& C & - & & - \\
Difference & FFA & 0.929 & - & -3.6330 \\
& OS & 0.430 & 0.6521 & \\
& Mean & -0.466 & -0.3333 & - \\
\hline & C & - & & \\
\hline
\end{tabular}


Table 3. (a) Comparison of C, FFA and OS with respect to pre, post and difference scores of Go to Pg in mm by one-way ANOVA test; (b) Pair wise comparison of C, FFA and OS w.r.t pre, post \& difference scores of Go to Pg in $\mathrm{mm}$ by Tukey's Honest Significant (HSD) Difference test procedure.

(a)

\begin{tabular}{ccccccc}
\hline Variable & Source of variation & Degrees of freed-om & Sum of squares & Mean sum of squares & F-value & p-value \\
\hline \multirow{3}{*}{ Pre treatment } & Between groups & 2 & 10.433 & 5.2167 & 0.1036 & 0.9018 \\
& Within groups & 42 & 2114.76 & 50.3516 & & \\
& Total & 44 & 2125.20 & & & \\
\multirow{5}{*}{ Post treatment } & Between groups & 2 & 365.733 & 182.8667 & 3.6020 & $0.0360^{*}$ \\
& Within groups & 42 & 2132.26 & 50.7683 & & \\
& Total & 44 & 2498.00 & & 37.012 & $0.0000^{* *}$ \\
& Between groups & 2 & 264.90 & 3.5786 & & \\
\hline
\end{tabular}

(b)

\begin{tabular}{ccccc}
\hline Treatment & Group & C & FFA & OS \\
\hline Pre treatment & Mean & 75.0000 & 75.7330 & 76.1670 \\
& C & - & & \\
& FFA & 0.9569 & - & - \\
Post treatment & OS & 0.8946 & 0.9848 & 82.2670 \\
& Mean & 75.6000 & 77.1330 & \\
& C & - & & - \\
Difference & FFA & 0.8266 & - & -6.1000 \\
& OS & $0.0368^{*}$ & 0.1316 & \\
& Mean & -0.6000 & -1.4000 & - \\
\hline
\end{tabular}

Table 4. (a) Comparison of C, FFA and OS with respect to pre, post and difference scores of FMA angle by One-Way ANOVA test; (b) Pair wise comparison of three groups (C, FFA and OS) with respect to pre, post and difference scores of FMA angle by Tukey's Honest Significant (HSD) Difference test procedure.

(a)

\begin{tabular}{|c|c|c|c|c|c|c|}
\hline Variable & Source of variation & Deg of freedom & Sum of squares & Mean sum of squares & F-value & $\mathrm{p}$-value \\
\hline \multirow{3}{*}{ Pre treatment } & Between groups & 2 & 183.67 & 91.83 & 3.79 & $0.0306^{*}$ \\
\hline & Within groups & 42 & 1017.43 & 24.22 & & \\
\hline & Total & 44 & 1201.11 & & & \\
\hline \multirow{3}{*}{ Post treatment } & Between groups & 2 & 254.17 & 127.08 & 3.51 & $0.0388^{*}$ \\
\hline & Within groups & 42 & 1518.8 & 36.16 & & \\
\hline & Total & 44 & 1772.97 & & & \\
\hline \multirow{3}{*}{ Difference } & Between groups & 2 & 18.23 & 9.11 & 0.47 & 0.6269 \\
\hline & Within groups & 42 & 810.96 & 19.30 & & \\
\hline & Total & 44 & 829.20 & & & \\
\hline
\end{tabular}

(b)

\begin{tabular}{ccccc}
\hline Treatment & Group & C & FFA & OS \\
\hline Pre treatment & Mean & 25.800 & 21.6000 & 21.433 \\
& C & - & & \\
& FFA & $0.041^{*}$ & - & - \\
Post treatment & OS & $0.040^{*}$ & 0.9954 & 23.533 \\
& Mean & 27.867 & 22.3330 & \\
& C & - & & - \\
Difference & FFA & $0.040^{*}$ & - & -2.100 \\
& OS & 0.131 & 0.8489 & \\
& Mean & -2.067 & -0.7333 & - \\
\hline
\end{tabular}




\section{4) Dentoalveolar changes: (Tables $\mathbf{5}$ and $\mathbf{6}$ )}

$\mathrm{C}$ group: U1 to $\mathrm{SN}$ angle indicated a reduction in proclination (mean diff. $12.9^{\circ}$ ) showing a highly significant change $(p=0.0018)$ and a statistically significant change was also seen with respect to L1 to MP angle (mean diff. $2.9^{\circ}$ ). It can be inferred that predominant amount of reduction in convexity was due to the intrusion and retraction of the upper anterior teeth and mild amount of lower incisor retraction had occurred.

FFA group: A highly significant change was seen with respect to reduction $\mathrm{U} 1$ to $\mathrm{SN}$ angle (mean diff. $13.6^{\circ}$ ), which was achieved partly by retracing and closing spaces in the proclined upper anterior teeth and could also be accounted to occur due to the distalizing effect of Fixed Functional Appliances. Mesialization of lower incisors had occurred, but it was statistically insignificant.

OS group: There was no significant difference with the pre and post-treatment values, so it can be inferred that the reduction in facial convexity was achieved predominantly by surgically correcting the underlying skeletal defect by advancing the mandible.

5) Soft tissue changes: (Tables 7 and $\mathbf{8}$ )

$\mathrm{C}$ group: Highly significant change $(\mathrm{p}=0.0052)$ was seen in the Nasolabial angle (mean diff. $-14.4^{\circ}$ ), indicating that overall reduction in facial convexity was on ac- count of retracting the upper incisors, which increased the nasolabial angle.

FFA group: An increase in the nasolabial angle (mean diff. $\left.-9.4^{\circ}\right)$ was observed which was statistically significant $(p=0.0062)$ and a highly significant $(p=0.0003)$ change was seen with respect to the mentolabial angle (mean diff. $-24.4^{\circ}$ ), which had increased post-treatment. These changes helped in the overall reduction of facial convexity.

OS group: Statistically significant change $(\mathrm{p}=0.0179)$ was seen in the nasolabial angle (mean diff. $-6.4^{\circ}$ ) which was increased and the angle formed from the soft tissue N'-Pg'-FH angle $(p=0.0107)$ had also increased (mean diff. $-2.3^{\circ}$ ). It can be inferred that there was significant change in the Mentolabial angle following the surgical correction of the underlying skeletal structure and in the nasolabial angle it was achieved by dentoalveolar changes.

6) Inference drawn from the VAS on the perception of treatment outcome (Graphs 1-3).

It was observed that the laypersons appreciated the changes in all the three groups equally.

The orthodontists and mostly the oral surgeons were more critical in evaluating the cases and this could be attributed to their enhanced observational skills regarding minute details for which they have been trained.

Table 5. Comparison of C, FFA and OS with respect to pre, post and difference scores of U1 to SN angle by One-Way ANOVA test.

\begin{tabular}{|c|c|c|c|c|c|c|}
\hline Variable & Source of variation & Deg of freedom & Sum of squares & Mean sum of squares & F-value & p-value \\
\hline \multirow{3}{*}{ Pre treatment } & Between groups & 2 & 720.8444 & 360.4222 & 3.0162 & 0.0597 \\
\hline & Within groups & 42 & 5018.800 & 119.4952 & & \\
\hline & Total & 44 & 5739.644 & & & \\
\hline \multirow{3}{*}{ Post treatment } & Between groups & 2 & 418.8444 & 209.4222 & 4.7009 & 0.0144 \\
\hline & Within groups & 42 & 1871.066 & 44.5492 & & \\
\hline & Total & 44 & 2289.911 & & & \\
\hline \multirow{3}{*}{ Difference } & Between groups & 2 & 1676.044 & 838.0222 & 4.6731 & 0.0147 \\
\hline & Within groups & 42 & 7531.866 & 179.3302 & & \\
\hline & Total & 44 & 9207.911 & & & \\
\hline
\end{tabular}

Table 6. Comparison of C, FFA \& OS w.r.t pre, post \& difference scores of L1 to MP angle by One-Way ANOVA test.

\begin{tabular}{|c|c|c|c|c|c|c|}
\hline Variable & Source of variation & Deg of freedom & Sum of squares & Mean sum of squares & F-value & p-value \\
\hline \multirow{3}{*}{ Pre treatment } & Between groups & 2 & 94.1778 & 47.088 & 0.5503 & 0.5809 \\
\hline & Within groups & 42 & 3594.133 & 85.574 & & \\
\hline & Total & 44 & 3688.311 & & & \\
\hline \multirow{3}{*}{ Post treatment } & Between groups & 2 & 510.9778 & 255.48 & 4.6104 & $0.0155^{*}$ \\
\hline & Within groups & 42 & 2327.466 & 55.415 & & \\
\hline & Total & 44 & 2838.444 & & & \\
\hline \multirow{3}{*}{ Difference } & Between groups & 2 & 259.7333 & 129.86 & 2.1361 & 0.1308 \\
\hline & Within groups & 42 & 2553.4667 & 60.7968 & & \\
\hline & Total & 44 & 2813.2000 & & & \\
\hline
\end{tabular}


Table 7. Comparison of C, FFA and OS with respect to pre, post and difference scores of Nasolabial angle by One-Way ANOVA test.

\begin{tabular}{|c|c|c|c|c|c|c|}
\hline Variable & Source of variation & Deg of freedom & Sum of squares & Mean sum of squares & F-value & p-value \\
\hline \multirow{3}{*}{ Pre treatment } & Between groups & 2 & 235.911 & 117.95 & 0.89 & 0.4148 \\
\hline & Within groups & 42 & 5512.66 & 131.25 & & \\
\hline & Total & 44 & 5748.57 & & & \\
\hline \multirow{3}{*}{ Post treatment } & Between groups & 2 & 48.577 & 24.288 & 0.19 & 0.8247 \\
\hline & Within groups & 42 & 5267.06 & 125.40 & & \\
\hline & Total & 44 & 5315.64 & & & \\
\hline \multirow{3}{*}{ Difference } & Between groups & 2 & 490.00 & 245.00 & 1.47 & 0.2404 \\
\hline & Within groups & 42 & 6976.80 & 166.11 & & \\
\hline & Total & 44 & 7466.80 & & & \\
\hline
\end{tabular}

Table 8. Comparison of C, FFA and OS with respect to pre, post and difference scores of Mentolabial angle by One-Way ANOVA test.

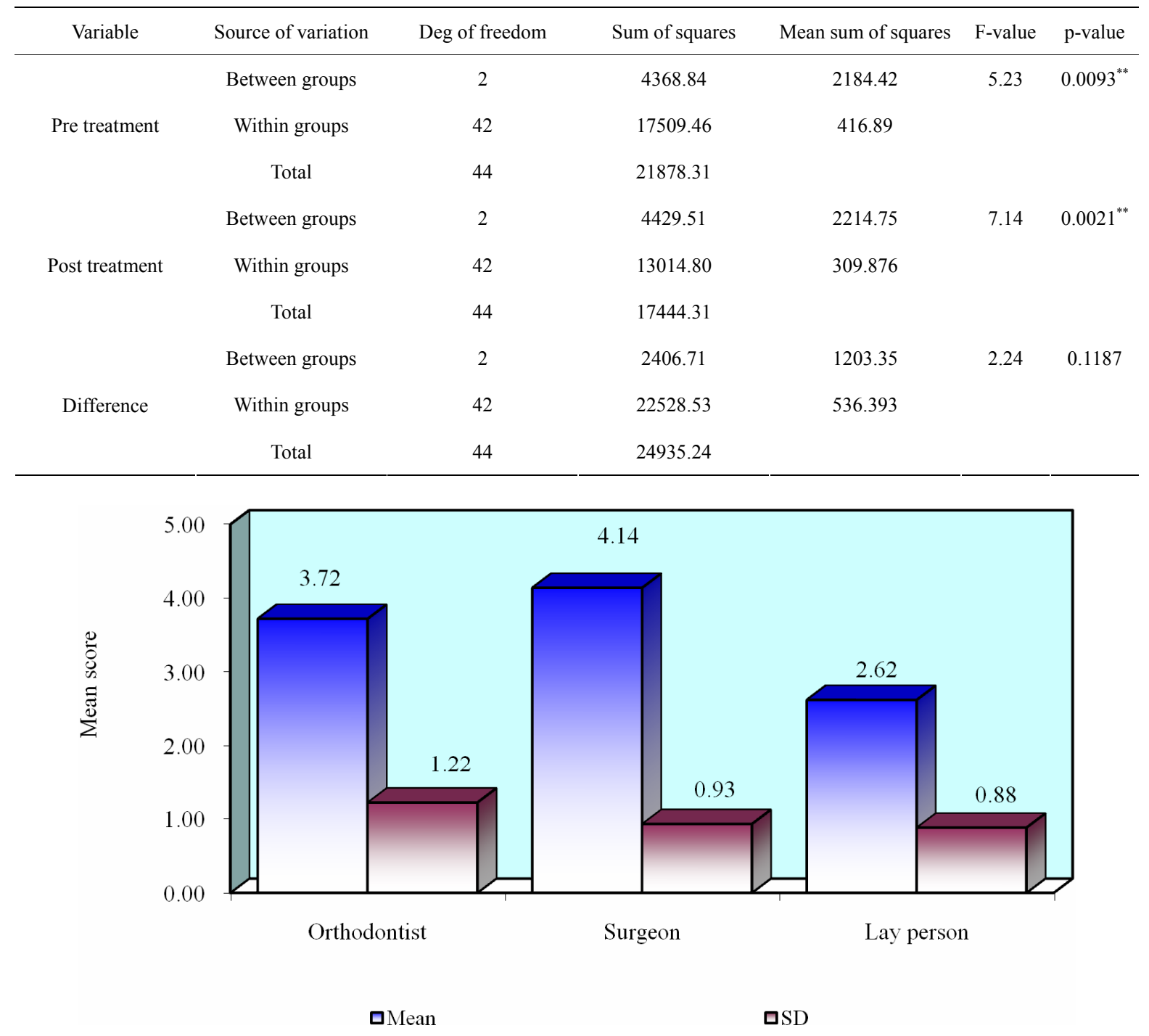

Graph 1. Comparison by VAS of Camouflage group. 


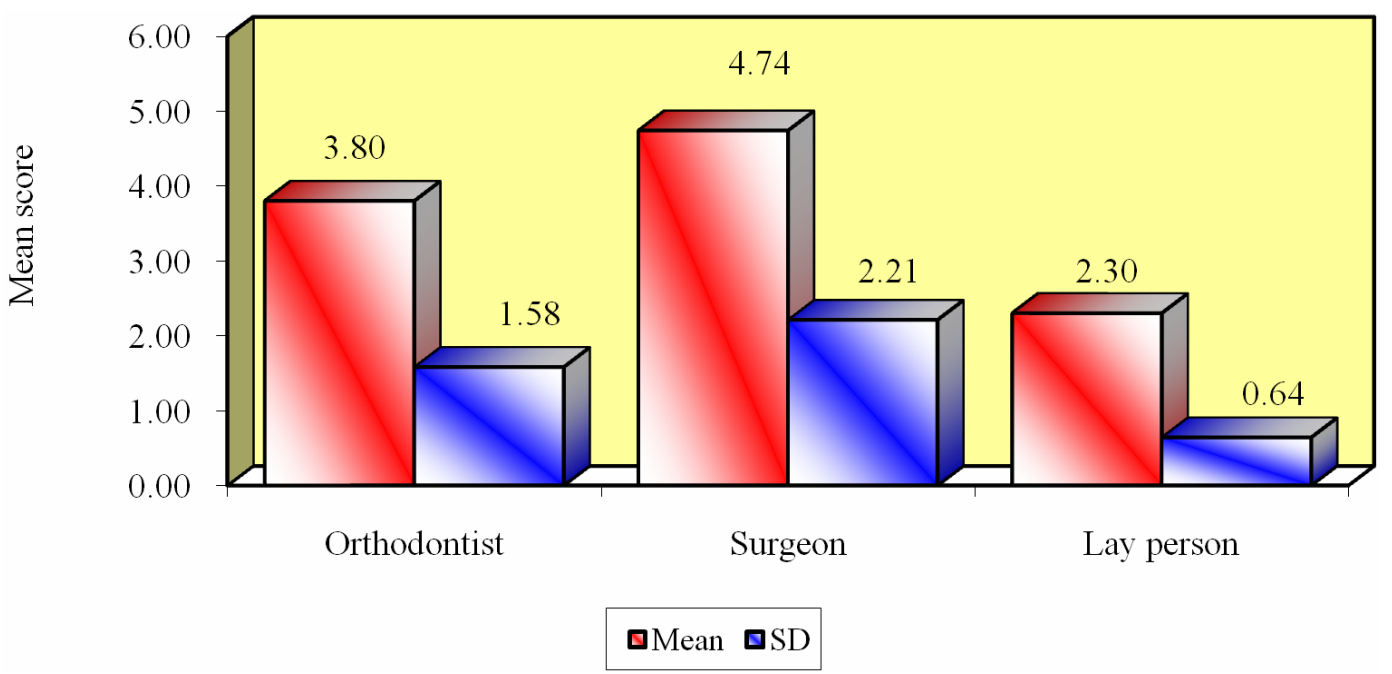

Graph 2. Comparisons by VAS of Fixed Functional Appliance group.

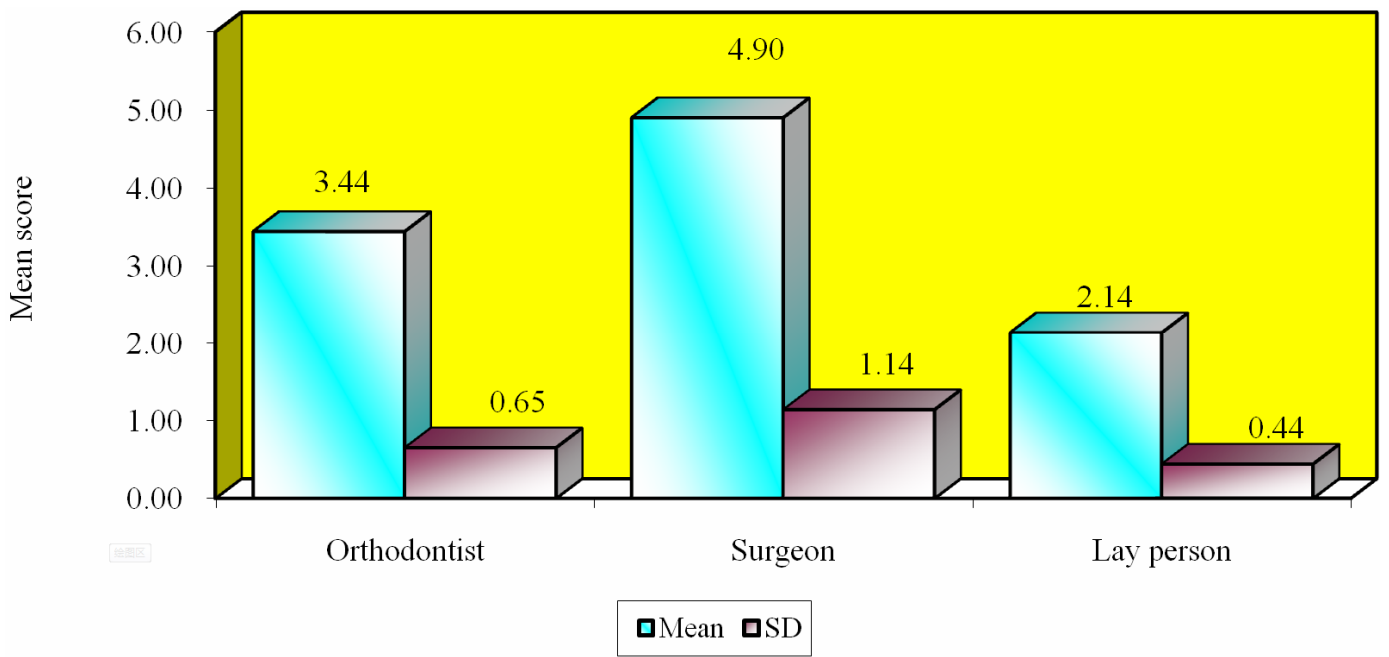

Graph 3. Comparison by VAS of Orthognathic Surgery group.

\section{DISCUSSION}

There are several studies comparing either of the two treatment modalities in young adult Class II patients [1416] but only one compares results of all the three treatment modalities [1].

Studies comparing camouflage orthodontic group (C group) vs orthognathic surgical group $[14,16]$, showed that the patients perception of facial improvement, posttreatment in both groups were significantly positive. In our study similar results were obtained on using a VAS. Although, the cephalometric analysis showed a highly significant change on the sagittal skeletal aspect only in the OS group with ANB angle (mean diff. 3.9 $9^{\circ}$.

There was a study, which compared the treatment induced changes in orthognathic surgical group (OS group) vs. fixed functional appliance group in adults [15], and a few others who campared the effect of FFA group in adult patients $[5,6,9,17]$. The overall results revealed that fixed functional appliances can be used as an alternative in borderline Class II patients.

A) Comparison of treatment changes between treatment groups:

1) Comparison between skeletal changes in groups:

The comparison of skeletal parameters between treatment groups, which includes changes in the sagittal relations, the position of the two jaw bases and the vertical positions have been included. One-way ANOVA test showed that there were no statistically significant differences between the treatment groups to start with. When the pre and post-treatment outcomes were compared, there was a highly significant change seen with the treatment outcomes.

Following which pair wise comparison of the three groups (C, FFA and OS) with respect to the pre and post-treatment values were compared by Tukey's Honest 
Significant Difference (HSD) test procedure, to compare each group against the other and to find out which among the three groups had significant changes when compared with each other.

In the sagittal plane, highly significant changes were seen in the OS group and the FFA group showed statistically significant changes. But there were no changes in the $\mathrm{C}$ group post treatment (Tables 1(a) and (b)).

In the vertical plane, when the pre and post-treatment relationship of the jaw bases was compared, it was shown that there were no changes in all the three categories. Since the growth pattern of the samples taken in this study, ranged from normodivergent to mild hyperdivergent growth pattern, there was no chances of worsening of profile due to the downward and backward rotation on the mandible (Tables 4(a) and (b)).

In the maxilla, all the parameters showed insignificant changes.

In the mandible, a highly significant difference existed in the OS group when compared with the FFA group, showing that there was a reduction in the overall facial convexity due to the advancement of the mandible, while an increase in the mandibular corpus length was also seen in the FFA group (Tables 2 and 3).

The dentoalveolar changes were observed in the upper incisors which was statistically significant in the $\mathrm{C}$ group in comparison with OS group, since dentoalveolar corrections were carried out in the $\mathrm{C}$ group and OS group correction was predominantly by surgical correction. In the lower incisor there was a significant difference present with the FFA group when compared with the $\mathrm{C}$ and OS group, this was because there was proclination of lower anteriors. However, the FFA group did not show a significant change when studied individually with respect to pre and post inclination of lower incisors (Tables 5 and 6 ).

Soft tissue changes had occurred in all the three groups, when compared with each other using the Tukey's HSD test, indicating that the three groups differed statistically with regards to nasolabial angle in all the groups and with respect to mentolabial angle was statistically significant in the OS group suggesting that the change was more pronounced in the surgical group (Tables 7 and 8).

B) Inference drawn from the VAS on the perception of treatment outcome (Graphs 1-3):

The changes in profile in the $\mathrm{C}$ group, was appreciated well by the laypersons (mean 2.62), followed by the Orthodontists (mean 3.72). There was a statistically significant similarity in the opinion amongst the orthodontists and laypersons. While the oral surgeons (mean 4.14) rated the treatment changes that occurred as "average".

The VAS scoring over the FFA group was perceived as "good" by the laypersons (mean 2.30) and by the orthodontists (mean 3.80). The Oral surgeons gave an "av- erage" score.

Whereas, no significant changes were appreciated in the OS group in contrast to the other groups when evaluated by all the three categories of raters in assessing the improvement in facial profile post treatment by laypersons (mean diff. 2.14), orthodontists (mean diff. 3.44) and oral surgeons (mean diff. 4.90) which was similar to the scoring in the other groups.

From the above findings it can be inferred that the laypersons appreciated the changes in all the three groups equally.

The orthodontists and mostly the oral surgeons were more critical in evaluating the cases and this could be attributed to their enhanced observational skills regarding minute details for which they have been trained.

\section{CLINICAL IMPLICATIONS \& CONCLUSIONS}

The aim of this study was to evaluate the effectiveness of each of the three treatment options (camouflage orthodontics, FFA and orthognathic surgery) on indiviuals from an Indian Population with a convex profile having boderline Class II malocclusion and derive their clinical implications.

- By camouflage orthodontics alone, reasonably favourable results were achieved. But care should be taken not to over-retract the upper anteriors, resulting in a unaesthetic appearance due to an increased $\mathrm{Na}$ solabial angle.

- The size and position of the nose should also be taken into account, to determine the extent of retraction that can be achieved. Patients with a large or upturned nose could be a limitation to camouflage orthodontics in Class II patients.

- There is limited possibility of retraction of upper anteriors in camouflage group when the cortical plate is thin.

- FFA carries out the correction by a combination of skeletal and dental changes. But long term studies with respect to stability needs to be evaluated.

- FFA has a tendency to procline lower anteriors; hence it should be ensured that the teeth are not proclined to start with.

- Significant improvement was observed in the Orthognathic surgery group, but the cost factor and the fear of undergoing surgery normally holds back patients from opting for this treatment modality. Hence, FFA could be considered as an alternative in borderline Class II adults.

- When the pre- and post-treatment profile photographs were assessed by laypersons and Orthodontists on a VAS, they rated all the three treatment modalities as "good", while, the Oral surgeons rated all the three groups as "average". 


\section{ACKNOWLEDGEMENTS}

The Authors are grateful to Dr. Dipesh D. Rao who helped them formulte the article.

\section{REFERENCES}

[1] Kinzinger, G., Frye, L. and Diedrich, P. (2009) Class II treatment in adults: Comparing camouflage orthodontics, dentofacial orhtopedics and orthognathic surgery-A cephalometric study to evaluate various therapeutic effects. Journal of Orofacial Orthopedics, 70, 63-91. doi:10.1007/s00056-009-0821-2

[2] Ruf, S. and Pancherz, H. (2004) Orthognathic surgery and dentofacial orthopedics in adult Class II division 1 treatment: Mandibular saggital split osteotomy versus Herbst appliance. American Journal of Orthodontics \& Dentofacial Orthopedics, 126, 140-152. doi:10.1016/j.ajodo.2004.02.011

[3] Conley, R.S. and Jerningan, C. (2006) Soft tissue changes after upper premolar extraction in Class II camouflage therapy. Angle Orthodontist, 76, 59-65.

[4] Demir, A., Uysal, T., Safi, Z. and Basciftci, F.A. (2005) Effects of camouflage treatment on dentofacial structures in Class II division 1 mandibular retrognathic patients. European Journal of Orthodontics, 27, 524-531. doi:10.1093/ejo/cji046

[5] Bock, N.C., Santo, C. and Pancherz, H. (2009). Facial profile and lip position changes in adult Class II, division 2 treated with the Herbst-multibracket appliance. A radiographic cephalometric pilot study. Journal of Orofacical Orthopedics, 70, 51-62. doi:10.1007/s00056-009-8801-0

[6] Konik, M., Pancherz, H. and Hansen H. (1997) The mechanism of Class II correction in late Herbst treatment. American Journal of Orthodontics \& Dentofacial Orthopedics, 112, 87-91. doi:10.1016/S0889-5406(97)70278-6

[7] Nalbantgil, D., Arun, T., Sayinsu, K. and Fulya, I. (2005) Skeletal, dental and soft tissue changes induced by the Jasper Jumper appliance in late adolescents. Angle Orthodontist, 75, 426-436.

[8] Pancherz, H. (1979) Treatment of Class II malocclusions by jumping the bite with the Herbst appliance. American
Journal of Orthodontics, 76, 423-442. doi:10.1016/0002-9416(79)90227-6

[9] Ruf, S. and Pancherz, H. (1999) Dentoskeletal effects and facial profile changes in young adults treated with the Herbst appliance. Angle Orthodontics, 69, 239-246.

[10] Pancherz, H., Ruf, S., Erbe, C. and Hansen, K. (2003) The mechanism of Class II correction in surgical orthodontic treatment of adult Class II division 1 malocclusions. Angle Orthodontics, 74, 800-809.

[11] Tsang, S.T., McFadden, L.R., Wiltshire, W.A., Pershad, N. and Baker, A.B. (2009) Profile changes in orthodontic patients treated with mandibular advancement surgery. American Journal of Orthodontics \& Dentofacial Orthopedics, 135, 66-72. doi:10.1016/j.ajodo.2007.01.033

[12] Phillips, C., Trentini, C.J. and Douvartzidis, N. (1992) The effect of treatment on facial attractiveness. Journal of Oral and Maxillofacial Surgery, 50, 590-594. doi:10.1016/0278-2391(92)90439-7

[13] Soh, J., Chew, M.T. and Chan, Y.H. (2006) Perception of dental esthetics of asian orthodontists and lay persons. American Journal of Orthodontics \& Dentofacial Orthopedics, 130, 170-176. doi:10.1016/j.ajodo.2005.05.048

[14] Cassidy, D.W., Herbosa, E.G., Rotskoff, K.S. and Johnston, L.E. (1993) A comparison of surgery and orthodontics in "borderline" adults with Class I division 1 malocclusion. American Journal of Orthodontics \& Dentofacial Orthopedics, 104, 455-470. doi:10.1016/0889-5406(93)70072-V

[15] Ruf, S. and Pancherz, H. (2004) Orthognathic surgery and dentofacial orthopedics in adult Class II division 1 treatment: Mandibular saggital split osteotomy versus Herbst appliance. American Journal of Orthodontics \& Dentofacial Orthopedics, 126, 140-152. doi:10.1016/j.ajodo.2004.02.011

[16] Tucker, M.R. (1995) Orthoganthic surgery versus orthodontic camouflage in the treatment of mandibular deficiency. Journal of Oral and Maxillofacial Surgery, 53, 572-578. doi:10.1016/0278-2391(95)90071-3

[17] Kinzinger, G. and Diedrich, P. (2005) Skeletal effects in Class II treatment with the functional mandibular advancer (FMA). Journal of Orofacial Orthopedics, 66, 469-490. doi:10.1007/s00056-005-0524-2 